\title{
Multiphase Flow Technology Impacts on Thermal Control Systems for Exploration
}

\author{
J. McQuillen*, J. Sankovic ${ }^{\dagger}$, and J. Lekan * \\ NASA John H. Glenn Research Center, Cleveland, OH, 44135
}

\begin{abstract}
The Two-Phase Flow Facility (T $\Phi$ FFy) Project focused on bridging the critical knowledge gap by developing and demonstrating critical multiphase fluid products for advanced life support, thermal management and power conversion systems that are required to enable the Vision for Space Exploration. Safety and reliability of future systems will be enhanced by addressing critical microgravity fluid physics issues associated with flow boiling, condensation, phase separation, and system stability. The project included concept development, normal gravity testing, and reduced gravity aircraft flight campaigns, in preparation for the development of a space flight experiment implementation. Data will be utilized to develop predictive models that could be used for system design and operation. A single fluid, two-phase closed thermodynamic loop test bed was designed, assembled and tested. The major components in this test bed include: a boiler, a condenser, a phase separator and a circulating pump. The test loop was instrumented with flow meters, thermocouples, pressure transducers and both high speed and normal speed video cameras. A low boiling point surrogate fluid, FC-72, was selected based on scaling analyses using preliminary designs for operational systems. Preliminary results are presented which include flow regime transitions and some observations regarding system stability.
\end{abstract}

\section{Introduction}

$\mathrm{T}$ wo phase flow and heat transfer is utilized within a multitude of different terrestrial applications ranging from electronic cooling to Heating Ventilation, and Air Conditioning (HVAC) to electrical power generation as a means of transporting heat energy by pumping and vaporizing a fluid. Different mechanisms are used to absorb and transport heat away from a source including pool boiling, forced convective flow boiling, spray cooling. The implementation of these mechanisms include thermosyphons; capillary flow devices such as heat pipes; vapor compression loops, such as refrigerators; and evaporative cooling towers.

Heat rejection levels range from tens of watts for individual electronic components to megawatts from projected space nuclear systems. The requirements for high thermal efficiency, combined with low mass and high reliability makes the design of space thermal control systems particularly challenging. The typical space systems requiring heat rejection include power systems, avionics, propulsion systems, (especially with cooling and pressure control within propellant tanks) and Environmental Control and Life Support Systems (ECLSS). For power ${ }^{1}$ and thermal ${ }^{2}$ systems where at least $100 \mathrm{~kW}$ need to be rejected, two-phase systems may be favorable based on a launch-mass basis (see Figure 1). For space systems where there is a relatively small temperature difference, two-phase systems have been implemented. For example, a capillary-pumped loop (CPL) provides heat transport between the cryocooler and radiator for the Near-Infrared Camera and Multi-Object Spectrometer (NICMOS) Cryo Cooler $(\mathrm{NCC})^{3}$ aboard the Hubble Space Telescope. Other spacecraft have also incorporated the use of heat pipes, loop heat pipes and capillary pumped loops.

\footnotetext{
* Aerospace Engineer, Fluid Physics and Transport Branch, , 21000 Brookpark Road, MS 77-5, Nonmember.

${ }^{\dagger}$ Radioisotope Power Systems Manager, Science Division, 21000 Brookpark Road, MS 142-5, Member.

$\$$ Launch and Mission Systems Manager, Constellation Systems Project Office, 21000 Brookpark Road, MS 77-7, nonmember.
} 


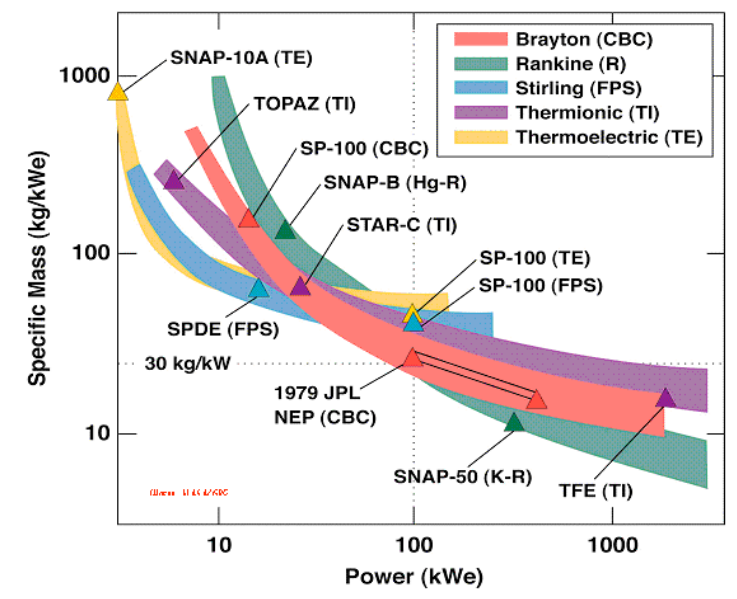

Figure 1. Trade Study Results for System Mass vs. Power Level via Different Power Levels (Mason)

These thermal management systems can be subdivided by their "functions" into three categories: Heat acquisition, heat transport, and heat rejection.

Heat can be acquired from several sources including power systems, such as the Rankine power cycle and fuel cells; machinery such as hydraulic pumps; electronics; and in the case of manned systems, from the life support systems. These heat sources may have their own heat transport and rejection components; however, the philosophy on larger spacecraft is to use only one or two, thermal transport loops to larger radiators. In this case, the heat sources can be arranged along the flow loop on parallel channels or in some serial fashion on a single channel. For single phase loops, heat is acquired as sensible heat, ie., the fluid temperature rises as the heat is absorbed. For two-phase systems, while some heat is absorbed by the fluid as sensible heat, the majority of the heat is absorbed as latent heat. Latent heat results in no temperature change of the fluid, but rather a phase change from the liquid to the vapor state. This can be accomplished via boiling or evaporation. Boiling and evaporation are influenced significantly by gravity primarily because of density difference between the vapor and liquid phases ${ }^{4}$, and consequently, vapor bubbles will tend to linger over hot surfaces and cause problems unless some other means are used to remove the hot fluid (in this case, the vapor bubble).

One method to remove the vapor bubble and thus the "hot fluid," in reduced gravity is to impose a flow across the heating surface. One critical parameter in boiling is the Critical Heat Flux (CHF). In this case, the rate of vaporization of the liquid is at or greater than the flow rate of liquid to replace the liquid that has been vaporized because the amount of heat per surface area of the flow channel is too great. Sustained operation at, or above the CHF, will result in dryout of the liquid film on the flow channel wall. This reduces the heat transfer coefficient (vapor cannot absorb heat as well as liquid) in the evaporator and may also lead to failure of the evaporator wall because of the localized thermal stresses. Boiling hysteresis, especially in highly wetting fluids, such as refrigerants, may affect where boiling occurs within the evaporator channel and may also result in similar thermal stresses being imposed on the heat transfer surfaces and may affect overall system stability..

The equipment used for heat transport function include the tubing length between the evaporator and heat rejection and the means of driving the flow, namely the pump or compressor. Pumps can be either active or passive, that is they have moving parts or not. The most commonly used passive means of pumping involves capillary wicks. Wicks are used in heat pipes, loop heat pipes (LHP) and capillary-pumped loops (CPL) and have limited capability in to drive the flows over great lengths through small diameter tubing. Consequently, these systems have been utilized in relatively low power situations where the heat source and sink are located close to each other. Porous wicks are sensitive to the presence of solid particles and bubbles that may occur either from non-condensable gasses or boiling within the wick.

Mechanical, or active, pumps can develop sufficient pressure to move the working fluid over large distances in small diameter tubing. However, there are issues involving pump lifetime reliability, especially since these thermal systems may be required to operate for years without any direct human maintenance or intervention. Some space systems are also sensitive to vibration because of either pointing or microgravity requirements the vibrations generated by the machinery and the moving fluid may cause problems. Finally, there are issues with regards to pump operation because of bubble ingestion. These bubbles can occur not only because all of the gas bubbles were not completely removed, but also because of bubble generation within the liquid. In space, the pressure of any closed loop system is always lowest at the pump inlet, and as such, this location is most susceptible to bubble evolution. Because of the localized saturated conditions, the liquid may vaporize or degas. Centrifugal pumps are the most susceptible to losing their prime, or pumping capability; however, even constant displacement pumps are susceptible as evidenced from the difficulties experienced by the ISS Elektron gear pump.

Heat pumps and refrigeration systems use compressors to drive the working fluid through the system. Compressors require lubrication in order to minimize wear and overheating. Containment and proper distribution of lubricating oils within compressors are problematic, but both sealed bearings and foil bearing are alternative 
methods for addressing the problem. Both bearing technologies have not yet been demonstrated for long-term reliability.

Ultimately, the heat from the thermal working fluid needs to be rejected to the environment by condensing the vapor back into a liquid. Within the condenser, drainage of the thermal working fluid is necessary in order to minimize the heat transfer resistance from the liquid condensate. "Shear-flow" condensers have been proposed as one means of keeping the liquid film moving by reducing the cross-sectional area of the flow conduit which would results in higher vapor velocities. However, the reduction in the cross-sectional area is usually no more than a factor of ten but the density difference between the phases is at least one hundred, so it is highly unlikely that the proposed mechanism is responsible for the limited success of this design. Further research is necessary before extending this concept from the prototype thermal management systems to larger, real systems.

External to the thermal fluid loop, the heat rejection to space can be done through two means: radiation or evaporation/sublimation of an additional, expendable fluid. The radiator size is responsible for the majority of the thermal management system mass and can be a significant factor in the size of the overall spacecraft as evidenced by the large radiator proposed for the former Jupiter Icy Moon Observation (JIMO) mission (Figure 2 ). The area required to radiate is a function of absolute

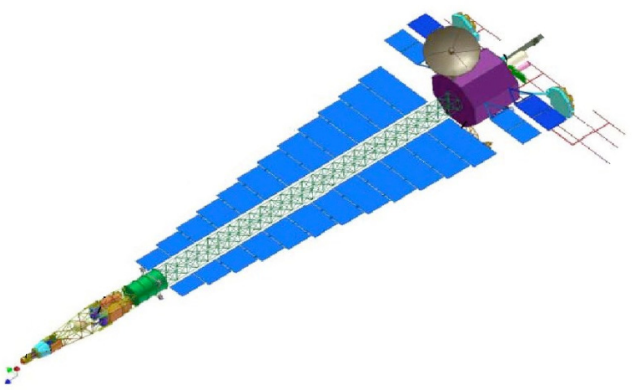

Figure 2. JIMO spacecraft conceptual configuration temperature of the radiator surface raised to the fourth power, thus even raising the radiator temperature by 10 to $20^{\circ} \mathrm{C}$, can have significant impact on the radiator size.

The use of expendable liquid for heat rejection by evaporation is the other means of rejecting heat to the space environment and is often selected for a variety of reasons:

- The environment is not conducive to radiation heat transfer, such as atmospheric operation during launch or entry phases of a mission

- The spacecraft is in an operational mode that is not configured to radiate heat, such as when the radiators are not deployed or open to space.

- "Topping" functions whereby the radiator is sized only to handle heat rejection loads that it will see for a majority of the time. This ability handles "peak" heat loads.

- The inability to predict or control where the radiator is pointing thus impacting the amount of heat that can be rejected. These types of systems are typically utilized on extra-vehicular activity (EVA) space suits.

The Apollo spacecraft and the EVA suits used sublimator technology ${ }^{5}$. In this technology, water is pumped into a porous media that is exposed to the high vacuum of space. The rapid vaporization freezes the water within the pores, thus limiting the discharge of excess water. Waste heat is used to warm the porous media which increases the rate of sublimation/vaporization of the ice. While this technology has been demonstrated to be effective and lightweight, the re-use of the sublimators require that the porous media prior undergo extensive cleaning or be replaced prior to reuse on subsequent missions. This is because solid impurities and mineral deposits clog the pores.

The Flash Evaporative System (FES) is utilized aboard the Space Shuttle to dissipate heat during descent and to "top" the heat rejection when the radiators can not provide enough cooling capacity. ${ }^{6}$ This system involves spraying water inside a heated cylinder and is capable of providing up to $30 \mathrm{~kW}$ of heat rejection. It has encountered problems with in-space freezing and system corrosion.

Ultimately, any space system that considers the use of multiphase flow for thermal management, must consider the following?

- Will the system work and over what range of operability?

- What causes instabilities in the system, what are the results of these instability, and how does the system recover from these instability events?

- How can the system be optimized?

While there are many different ways to acquire, move, and release heat that involved multiphase flow, there are also several common fundamental concepts. As a result, by developing a sufficient understanding of these concepts, the application of this understanding would have far-reaching benefits. 


\section{The Multiphase Flow Technology Program}

In 2004, the NASA Headquarters organization formerly known as the Office of Biological and Physical Research initiated fundamental reduced gravity two-phase flow research directed at addressing NASA's technology needs for future space mission. In 2004, the Office of Exploration also initiated Project Prometheus to examine the use of nuclear power to enable electric propulsion for research platforms with high power available also for science instrumentation. The Rankine power cycle was considered for further investigation over the Brayton and Stirling power cycles because it was the only power cycle that could provide the projected power requirements and low system mass while minimizing the temperature difference between the reactor and the radiators. ${ }^{1}$

NASA estabilished an effort to focus on addressing the enabling multiphase issues for the operation of a liquid metal Rankine cycle. A NASA Strategic Working Group was formed to address these issues. The findings by a team of specialists in nuclear energy, heat transfer and multiphase flow technology (Lahey and Dhir ${ }^{8}$ ) identified near-term key technology issues and a long term research and development program to qualify a Rankine power cycle for use in space. This effort would also develop the necessary design tools for all two-phase systems, regardless of their application, and included model development, phasic point measurements, and model verification.

While the focus of this working group was on the Rankine power cycle, the only real difference between this power cycle and thermal management systems is that a turbine is incorporated into the two-phase flow loop in order to convert the flow momentum into electrical energy. There are many similarities in the issues faced by both the Rankine power cycle and the two-phase thermal management systems.

The Multiphase Flow Technology Program was focused on four technical thrusts, namely passive phase separation, gravity-independent phase change, the thermal hydraulics of boiling, and system stability.

\section{A. Phase Separation}

Phase separation is necessary in order to avoid bubble ingestion into pumps that would result in the loss of pump priming. While cavitation may still occur pending the degree of thermal and dissolved gas saturation within the liquid, bubbles ingested into the pump stall centrifugal pumps because of gas bubble occlusion at the pump inlet. Positive displacement pumps are unable to maintain sealing, and hence their suction, around their gears. Furthermore, in the case of high-quality or high void fraction flows, liquid droplets impinging on either compressor surfaces or turbine blades will erode the surfaces of these components leading to failure because of premature wear.

Phase separators can also be used to enhance efficiency and reduce uncertainty due to two-phase flow distribution in parallel channels. Flow and phase distribution within manifolds is significantly affected by gravitational levels. Phase separation and the subsequently feed of only a single phase into parallel channels can remove much of the uncertainty associated with this known two-phase instability.

"Active" phase separation requires power and moving component in order to achieve the desired phase separation. Motor-driven, centrifugal separators are an example of active separators that rely on the rotation to develop a centrifugal acceleration to generate an equivalent hydrostatic head to separate the phases based on their density differences. Passive techniques typically utilize flow momentum or capillary forces to achieve their desired end. Usually, active techniques require additional power and rotating machinery. This activity was later reassigned programmatically to support waste water reclamation within the Advanced Life Support Program..

\section{B. Gravity-Independent Phase Change}

Determination of the range of conditions for gravity-independent phase change enables the ability to test the actual subsystem and/or components in normal gravity and have confidence that similar performance will be achieved in reduced gravity. The flow may be inertial-based (Mudawar, et al. ${ }^{9}$ ) or capillary driven. Mudawar has identified that liquid velocities of at least $1.5 \mathrm{~m} / \mathrm{s}$ will produce similar looking flow regimes in reduced gravity and in normal gravity at all orientations of the flow channel with respect to gravity. Other means, such as pressuredriven flow into a packed bed ${ }^{10}$, also have shown promise towards operation in any gravity level. Another method that is proposed is the use of twisted tape inserts as a means to induce a secondary centrifugal flow within the evaporator or boiler.

\section{Thermal Hydraulics Of Boiling}

As was stated earlier, research into flow boiling in reduced gravity has been extremely limited and been conducted primarily at relatively low qualities. As such, it is necessary to quantify the thermal hydraulics within the boiler/evaporator in order to increase the design reliability and identify potential thermal hydraulic transients that influences the boiler output. No research to date has been conducted in reduced gravity at higher qualities. The 
mechanisms governing boiling and or evaporation in the thin liquid films of annular flow affect liquid droplet entrainment into the vapor core and will be undoubtedly different in reduced gravity as opposed to normal gravity. The liquid film dryout and rewetting should also exhibit differences in behavior and will impact the thermal stresses encountered by the heat transfer surfaces.

Similarly, flow regime transition boundaries, especially if transition location varies significantly with time along the boiler axis, will impact the local heat transfer coefficients and thus the local thermal stresses encountered by the boiler wall. This occurs in the transition from single-phase liquid to bubbly flow, to slug flow, to annular flow, to droplet flow or mist flow to vapor flow.

\section{System Stability}

In the past, NASA has been concerned with system stability, but primarily from the standpoint of difficulties associated with system startup, shutdown, and step-changes in system set point operations. However, Lahey and Dhir point out that there are numerous instability mechanisms that are inherent with the system simply being twophase. Among these instability mechanisms are pumped loop instability, parallel channel instability, density wave oscillations, and critical heat flux. These have been encountered, identified and accounted for in normal gravity systems and are conjectured to likely occur in reduced gravity.

\section{E. Approach}

These four area discussed above would be addressed via complementary efforts using ground-based testing, NASA's C-9 low gravity research aircraft, an ISS thermodynamic test-bed facility (designated as T FFy--TwoPhase Flow Facility) and diagnostic development and modeling. Ground-based and research aircraft testing was to focus on two-phase issues related to specific components, such as evaporators and make preliminary measurements of two-phase flow attributes, such as liquid film thickness, droplet size and velocity distributions.. The ISS test-bed was to be integrated within the Fluids Integrated Rack (FIR). The objectives of the ISS test-bed's included a demonstration of a long duration, low gravity, single fluid, multiphase flow thermodynamic system and to examine two-phase instability phenomena including startup, shutdown and other critical behaviors. Diagnostics are being developed in order to measure these critical two-phase flow attributes and phenomena that are necessary to develop and verify models that would extend the fundamental knowledge to other designs and systems.

\section{T FFy C-9 Rig}

The initial purpose of test rig is to determine the range of gravity independent flows, not only as a function of fluid conditions in terms of sub-cooling, heat transfer, flow rates, but also evaporator geometry. Initial test sections include a straight, empty, $6 \mathrm{~mm}$. inner diameter (ID) tube and a $6 \mathrm{~mm}$ tube with a twisted tape insert. Future testing will include using evaporatons with different geometries such as a rectangular cross section.

The rig will also be used to demonstrate the new diagnostic techniques that are necessary to measure critical phasic parameters, such as droplet or bubble size and velocity, turbulence and liquid film thickness. Depending on the sensitivity of some of these measurements to the residual gravity level aboard the aircraft and the time duration needed to make these measurements, it is intended to make several of these measurements utilizing this rig.

Finally, the hardware will be used to conduct preliminary assessments of system stability that can be used to map the test matrix for the ISS T FFy stability experiments.

\section{F. Rig Description}

The T FFy C-9 experiment is a dual closed loop, two-phase phase change rig. The flow schematic is illustrated in Figure 3. 


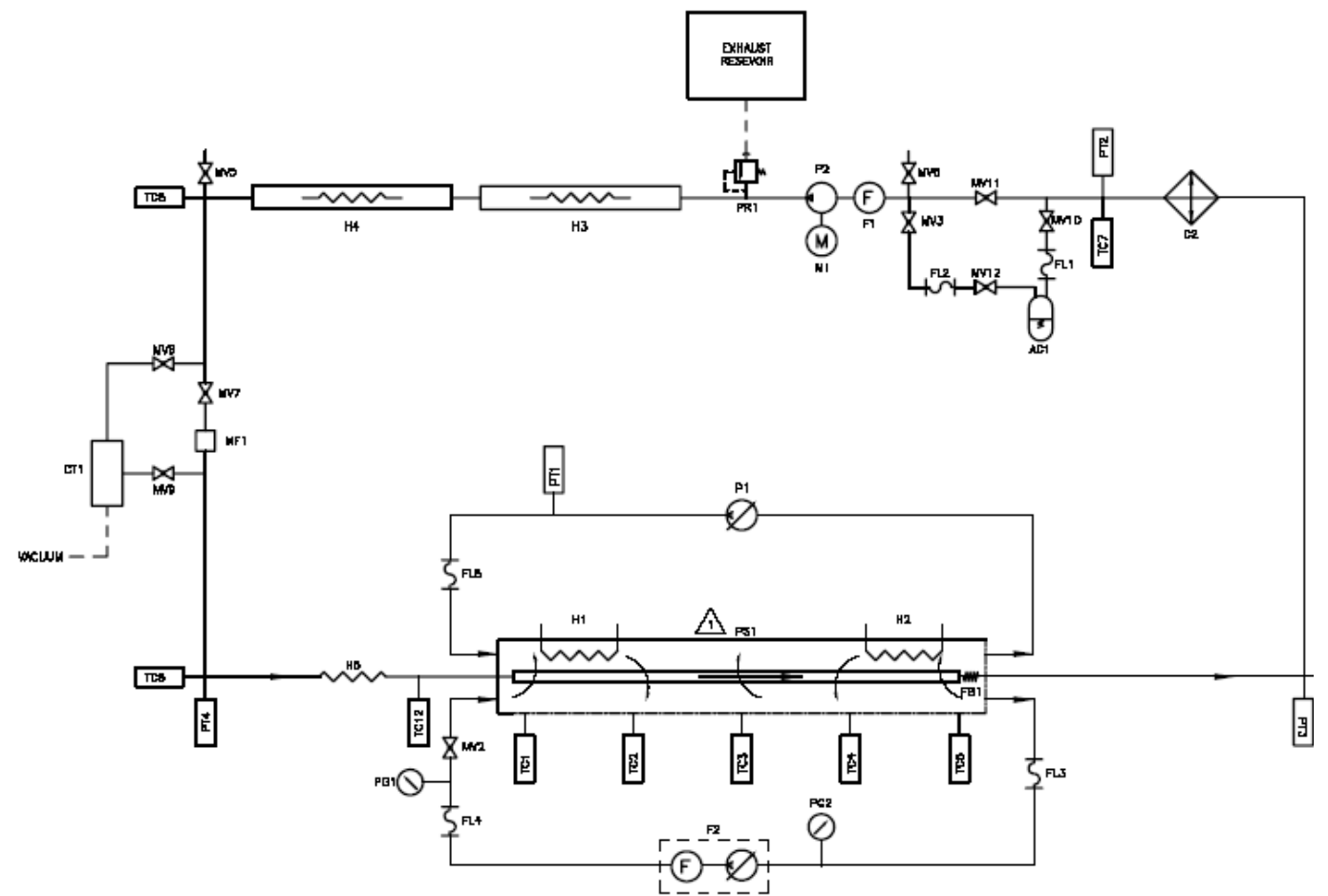

Figure 2. T FFy C-9 Rig Flow Schematic

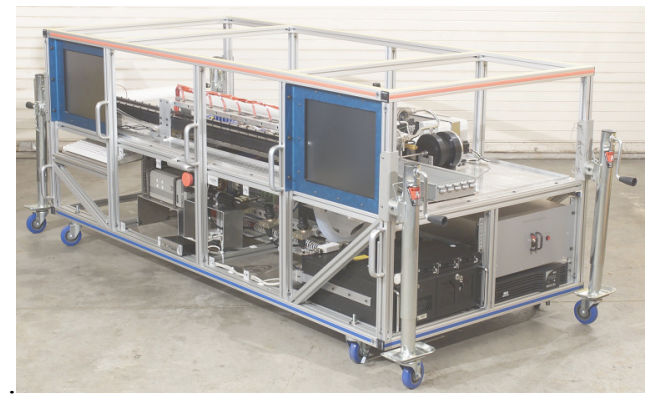

Figure 3. T FFy C-9 Rig

The rig is constructed from structural channel and aluminum shelves that provide accommodation for the experiment's infrastructure (see Figure 4). The experimental rig is aircraft $2.4 \times 1.1 \times 0.9 \mathrm{~m}$ and weighs $295 \mathrm{~kg}$. A removable plate houses both flow loops and all of fluid components are mounted at the same horizontal level in order to minimize the fluctuating hydrostatic pressure changes associated with the aircraft's parabolic flight profile. Power and high-speed video umbilicals are routed from the various rack-mounted subsystems in the base and on each end of the payload. The outer loop uses hot water to drive boiling and evaporation within the second flow loop that utilizes FC-72, a 3M Fluoroinert that is a mixture of several perfluorohexane isomers. The FC-72 boils at $56^{\circ} \mathrm{C}$ at one atmosphere. The water temperature is kept below its boiling point. The phase change occur within a tube-inshell heat exchanger boiler whereby the internal quartz tube has an internal diameter of $6 \mathrm{~mm}$. and the shell is a 0.91 $\mathrm{m}$ long aluminum extrusion $(2.5 \times 10 \mathrm{~cm})$, with glass windows.

Two fluid pumps circulate the flow within their respective flow loops. Condensation of the FC-72 vapor occurs within an air-cooled condenser and the flow is recirculated back to the tube-in shell heat exchanger. A bellows is utilized to account for expansion of the FC-72 due to density changes associated with the rise in liquid temperature and the phase change. The bellows is driven via a stepper-motor that is controlled via the data acquisition and control program.

Water loop temperature can be set to a maximum value of $95^{\circ} \mathrm{C}$ which drives the amount of heat transferred into the FC-72 flow loop. The FC-72 flow loop can be pressurized from 10 to 21 psia. The FC-72 flow rate can be set from 2 to $14 \mathrm{~g} / \mathrm{s}$. Typically, the condenser outlet temperature is controlled to be $9^{\circ} \mathrm{C}$ less than the saturation temperature corresponding to the system pressure. This is to eliminate cavitation within the pump while minimizing the duty cycle for the FC-72 preheaters.

For each flow loop, the principle measurements will include flow rates; fluid and wall temperatures, especially along the length of the test section; pressures, both absolute and differentials across the test section length and the pump, and void fraction. High speed video will be used to provide both an assessment means of flow regime and 
their transitions along the length of the test section. The high speed video will also provide an initial qualitative assessment as to the "quality" of the phase change by detecting liquid droplets at the test section exit.

The high-speed video system is the NAC HSV-500. This system utilizes a SVHS-video tape to record data and can be controlled remotely by the data acquisition system. The system records images that are $640 \times 450$ pixels. The system is time synchronized to the data acquisition and control system via IRIG-B

Four Silicon Video 1281 CMOS cameras and frame grabber were integrated to an additional data acquisition system which records an image of $1280 \times 64$ pixels from each camera. These cameras can normally acquire images of $1280 \times 1024$ pixels at 30 images per second. The four images are recorded into a single TIF file, but can be acquired at a much faster rate, up to 250 images per second. Figure 5 shows a typical image acquired by this system.

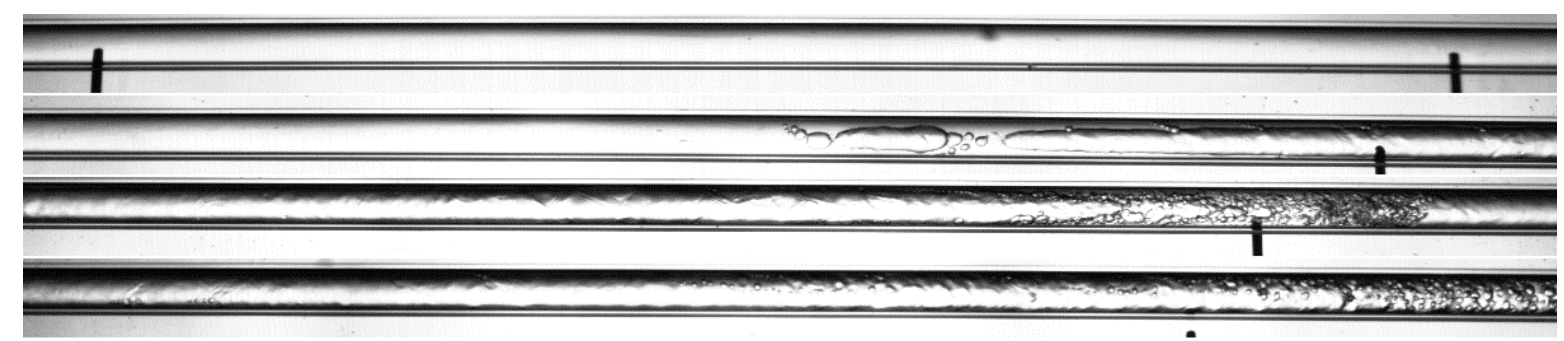

Figure 4. Representative Image Stored By Silicon Video Cameras Showing Evolution From Heating Subcooled Liquid To Bubble To Slug To Annular Flow

The data acquisition and control system uses a Labview ${ }^{\mathrm{TM}}$ application to set flow rates, system pressures, and temperatures at various points within the flow loops, and it also controls the NAC high speed video system. System status is displayed on a Graphical User Interface (GUI) that approximates the FC-72 flow loop schematic. Additional GUI's are used for setting control parameters and displaying them, setting parameters for closed loop controller functions.

A separate ground-support cart was assembled to service the fluid loops on the rig. Quick disconnects were used as umbilicals between the rig and the service cart. Filling or charging the loops to appropriate fill levels through a filter to capture particulate matter was possible. Semipermeable membranes were used to "degas" the fluids. This was necessary not only to avoid confusing degassing with boiling phenomena, but to minimize the experiment's overall power consumption. Without degassing the fluid, additional liquid sub-cooling was necessary at the condenser outlet to avoid pump cavitation due to the bubble formation at the pump inlet. The water flow loop was degassed primarily to avoid bubble formation within the shell and tube heat exchanger that would obscure the visualization within the FC-72 evaporator.

\section{G. Procedure}

The water loop served as the heat source for the FC-72 and the water temperature was set to 70,80 or $90^{\circ} \mathrm{C}$. As the water was being thermally conditioned, the pressure on the FC-72 loop was set to 20, 17 or 14 psia. The condenser outlet temperature was set to $10^{\circ} \mathrm{C}$ lower than the saturation temperature for the loop pressure, or 55,48 or $44^{\circ} \mathrm{C}$ respectively. This was to avoid pump cavitation. The fluid preheater was adjusted to provide liquid at a liquid sub-cooling of $9^{\circ} \mathrm{C}$ respective to the system pressure and to account for any heat losses to the ambient between the condenser and the test section inlet. While the liquid sub-cooling could have been raised closer to the temperature value for saturation, it was not because of the desire to limit vaporization to the flow visualization section, ie., the evaporator. Otherwise, pressure excursions or changes in the flow rate set point could have initiated boiling in the preheater section.

The FC-72 flow rates were adjusted from 3 to $11 \mathrm{~g} / \mathrm{s}$. Data was gathered on average for at least two minutes or three "periods" of the sinusoidal pressure oscillations that were observed at the higher water bath temperatures.

After the system had "stabilized" at the new set points, both the NAC high speed video system and the four Silicon Valley cameras acquired data. In order to synchronize the data between the two camera systems, the LED backlighting was disabled momentarily and then re-enabled. The Silicon Valley cameras acquired until the memory buffer was filled. The NAC system was then stopped.

At any given condition, the FC-72 flow rates were first adjusted. After a series had been completed, then the system pressure was adjusted. Finally, the water bath temperature was the last parameter to be altered.

\section{H. Observations}


Flow boiling was easily initiated within the evaporator. All three of the standard flow regimes were observed: bubble, slug, and annular as well as the transitional flow regimes. There was some degree of stratification observed, especially at the slower flow rates. This was easily distinguished as the boiling nucleation site fed its bubbles into a large vapor mass that grew upstream of the nucleation site (see Figure 6). For this test condition, there was no bubble nucleation prior to this site.

As was stated earlier, bubble nucleation can be problematic if the location varies significantly. For the most part, preliminary data analysis indicates that when the bath temperature was at either 80 or $90^{\circ} \mathrm{C}$, boiling was initiated at or near the entrance for the test section.

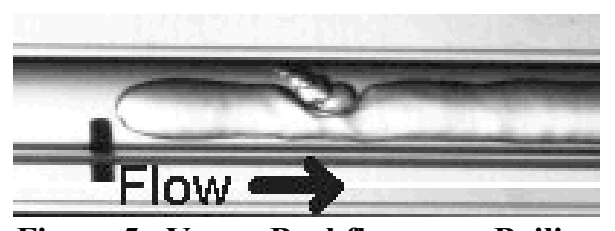

Figure 5. Vapor Backflow near Boiling Nucleation Site.

This was not the case for when the bath temperature was at $70^{\circ} \mathrm{C}$. While in Figure 7, there appears to be a weak link between the appearance of the first nucleation site and both the flow rate and the system pressure, it should be remembered that prediction of heterogeneous bubble nucleation sites on smooth tubing is still relatively unreliable and certain techniques to manufacture these sites into the

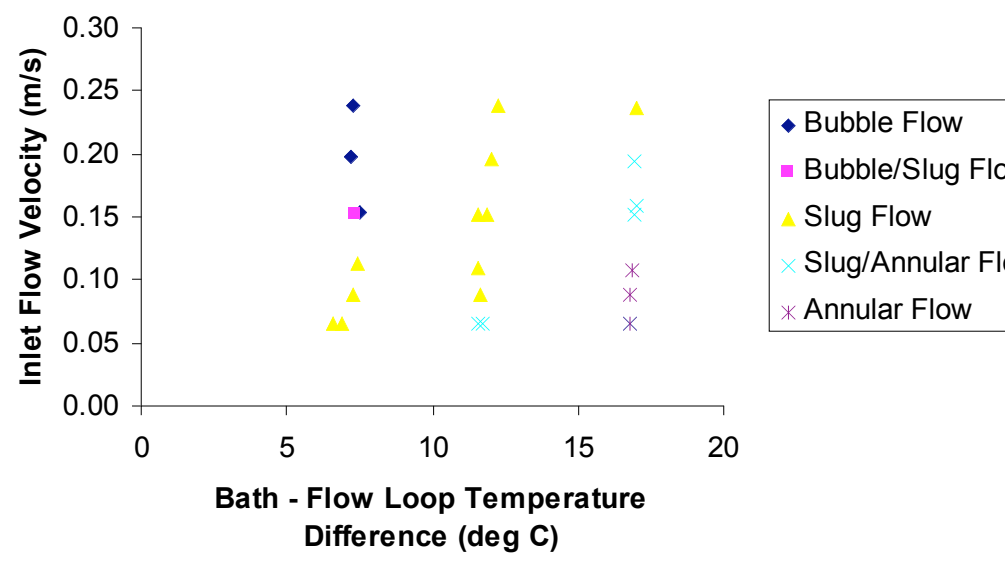

Figure 7: Flow Regime Map for Bath Temperature $=70^{\circ} \mathrm{C}$

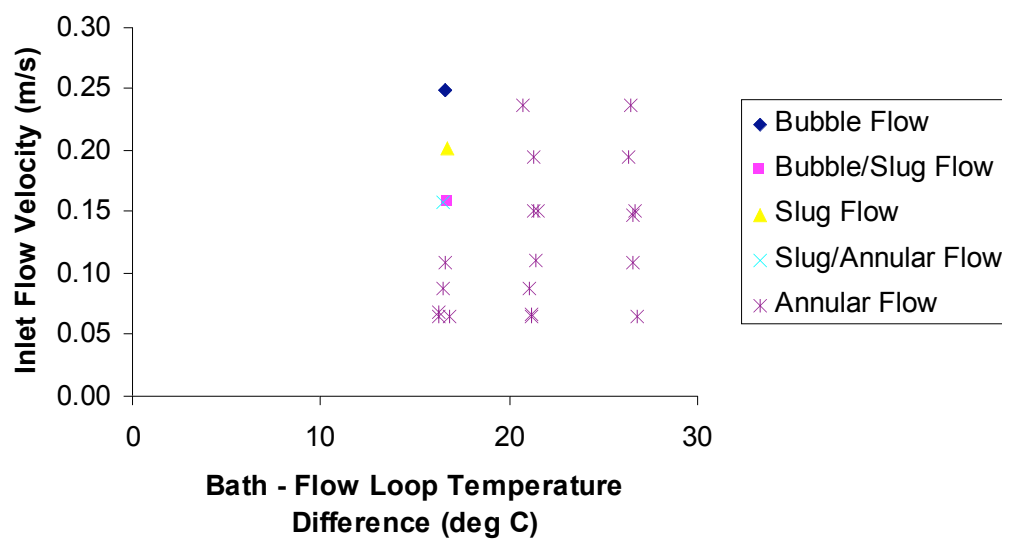

Figure 8. Flow Regime Map for Bath Temperature $=80^{\circ} \mathrm{C}$

surface to fix the axial location should be used.

Vapor bubble growth appeared to occur from three different mechanisms: Bubble nucleation and coalescence into larger bubbles, liquid evaporation, either as a means of superheated liquid film evaporation, and bubble expansion due to the pressure loss along the evaporator because of either ideal gas expansion or change in the local liquid-vapor saturation conditions.

While it was highly desired to achieve liquid dryout within the evaporator, it was not observed under the test conditions. Figures 7 and 8 are preliminary "flow regime maps" for the observed outlet flow regimes with bath temperatures of 70 and $80^{\circ} \mathrm{C}$. Data for the bath temperature of $90^{\circ} \mathrm{C}$ as only the annular flow regime was observed with larger temperature differences than those seen in Figure 8 and 9 and is consistent with this data.

As was indicated earlier, the T FFy C-9 Rig had multiple individual closed-loop control loops. These loops controlled heaters for the water bath and the

FC-72 inlet temperature. They also controlled the fan speed on the FC-72 condenser based on the outlet temperature from the condenser. Another loop controlled the FC-72 pump speed based on input from the flow meter. Finally, a stepper motor drove a bellows to control the system temperature utilizing a proportional control algorithm. 
First Nucleation Sites

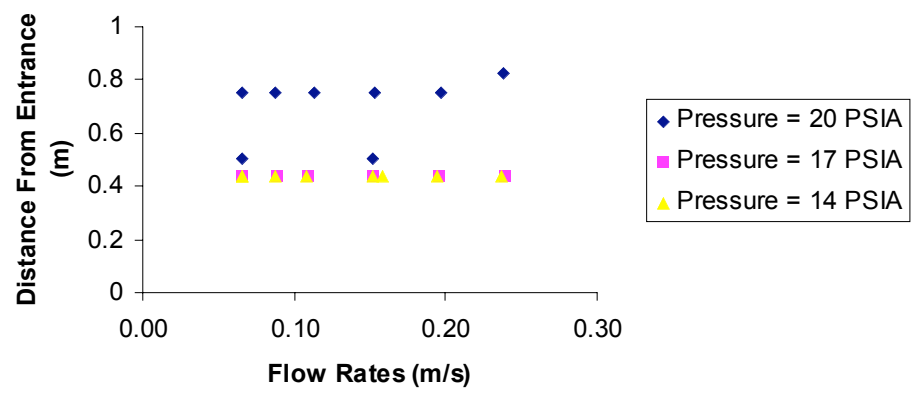

Figure 9. Axial Distance of Boiling Nucleation Sites for Bath Temperature $=7^{\circ} \mathrm{C}$
For the most part, the various controllers performed well at all conditions, but there are some stipulations to this statement. First, the water bath temperature controller would cycle on and off with a bandwidth of approximately $\pm 0.5^{\circ} \mathrm{C}$. This could be seen in Figure 10, the time traces for the five thermocouples placed alongside the evaporator in the water bath. Note that the approximate cycle time appear appears to be about 18 seconds.

When the bath temperature was set to $70^{\circ} \mathrm{C}$, the other parameters were well behaved with regards to the set points. At the higher bath temperatures of 80 and $90{ }^{\circ} \mathrm{C}$, fluctuations appeared usually in the pressure measurements and sometimes within the temperature measurements. The FC-72 flow rate held constant. As evidenced by the series of time traces shown in Figure 11, the cycling time for these oscillations were about 45-50 seconds, much slower than those associated with water bath temperature. With the exception of the evaporator inlet temperature, the pressure and temperature for the FC-72 loop appear to rise and fall nearly simultaneously.

There was no apparent relationship between the oscillations in the FC72 loop and the water bath temperature.

The synchronization between these sensors can be explained by momentary increases in the evaporation rate. While the bellows used for pressure control had a much larger volume than both the volume of the evaporator and condenser, the proportional control algorithm did not adequately address this slow

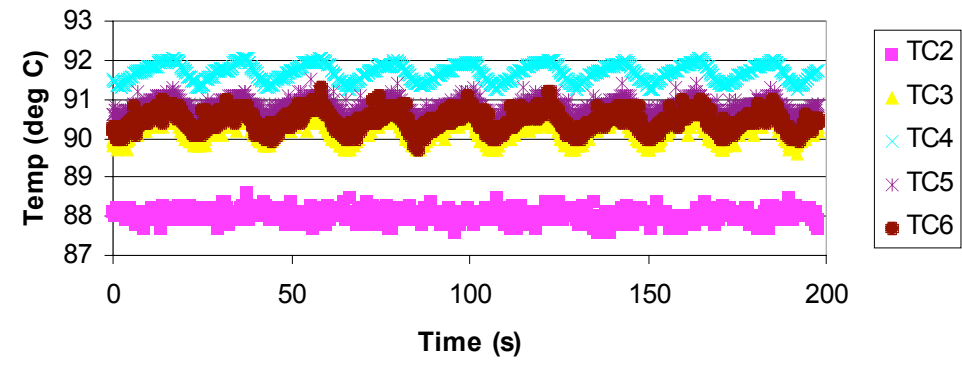

Figure 10. Typical Water Bath Temperature Time Trace

rising pressure oscillation. As a result, as the evaporation rate slowly increased, the pressure would rise within the entire flow loop volume. This raised the saturation pressure and temperature within the flow loop. As a result, the latent heat transfer slowed, but there was still sensible heat transfer occurring as evidenced by the fluid temperature rise in the evaporator.

A recommendation for future testing is to incorporate a Proportional Integral Derivative (PID) control scheme for the pressure control system instead. 


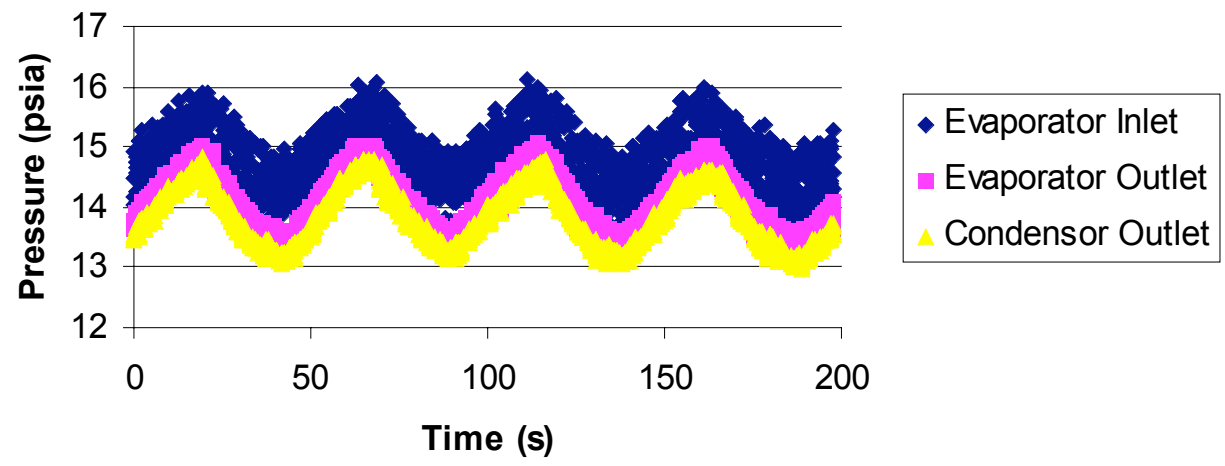

(a)

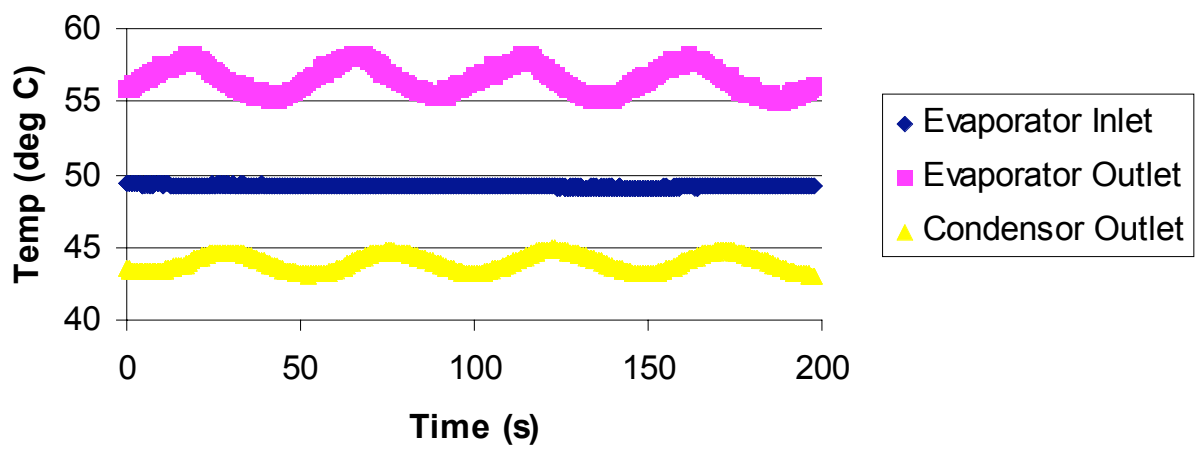

(b)

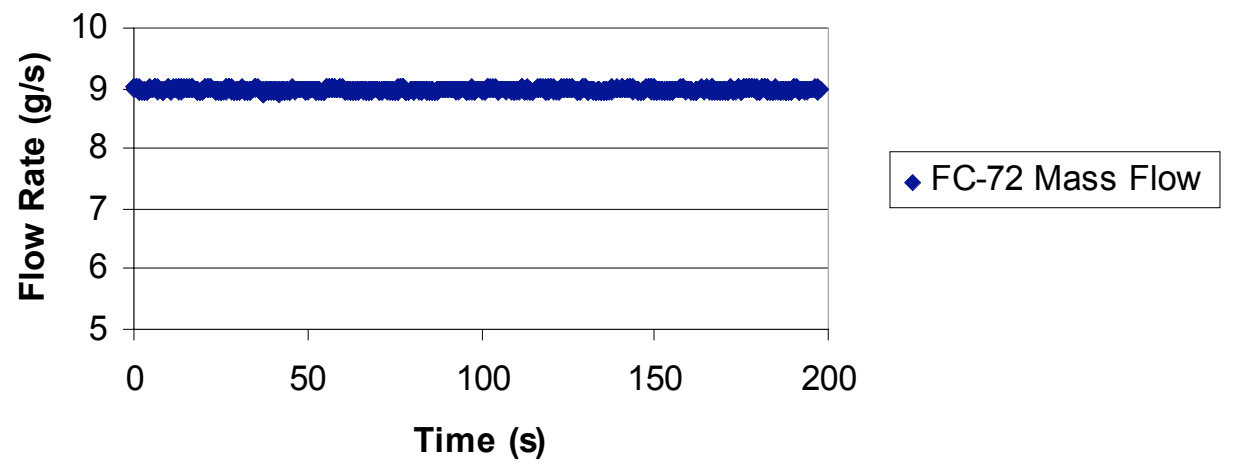

(c)

Figure 11. Typical Time Traces for Water Bath Temperature $=90^{\circ} \mathrm{C}$ (a) FC-72 Loop Pressures, (b) FC-72 Loop Temperatures, (c) FC-72 Mass Flow Rate

\section{Conclusion}

The T FFy C-9 Rig has been constructed and preliminary tests in a normal gravity environment have demonstrated the rig's potential in obtaining useful data with regards to studying the effects of evaporator geometry on establishing gravity independent boiling conditions that may be encountered in shell and tube heat exchangers 
and boiling nucleation studies, In addition, initial tests have demonstrated the existence of a sinusoidal pressure and temperature fluctuation within the system that should be controllable. Further testing, with a twisted tape insert in both normal and reduced gravity environments will be conducted and the results will be analyzed.

This rig will be a useful asset in examining component level behavior in two-phase thermal management systems.

\section{Acknowledgments}

The authors acknowledge the technical contributions of Andrew Sexton, Dwayne Kiefer, Robert Skupinski, Elizabeth Gray, Bill Birchenough, Arthur Stachowicz, Dawn Sgro from QSS, Inc. for the design, assembly, checkout, and testing of the rig. Further acknowledgments are due Mark Wernet from the NASA Glenn Research Center and Tony Opalski from QSS, Inc. for the development of the four camera Silicon Video system. Kirk Logsdon from the NASA Glenn Research Center is recognized for the oversight and coordination of the C-9 rig design and assembly. Finally, Fran Chiaramonte and the Human Systems Research and Technology Division in the Exploration Systems Mission Directorate at NASA Headquarters are acknowledged for their support.

\footnotetext{
${ }^{1}$ Mason, L. S., "Power Technology Options For Nuclear Electric Propulsion," Proceedings of the International Energy Conversion Engineering Conference, Paper No. 20159, 2002.

${ }^{2}$ Ungar, E., "Single phase vs. two-phase active thermal control systems for space applications - A trade study," AIAA-1995-634,, 1995

${ }^{3}$ Jedrich, N., Gregory, T., Zimbelman, D., Cheng, E., Petro, L., Cottingham, C., Buchko, M., Kaylor, M., and Dolan F., "Cryogenic cooling system for restoring IR science on the Hubble Space Telescope," Proceedings of SPIE -- Volume 4850, IR Space Telescopes and Instruments, , pp. 1058-1069, 2003

${ }^{4}$ Kim, J., "Review of Reduced Gravity Boiling Heat Transfer: US Research," J. Jpn. Soc. Microgravity Appl. Vol. 20, pp., 264-271, 2003.

${ }^{5}$ Gillen, R. J., Brady, J. C., and Collier, F., "Apollo Experience Report: Lunar Module Environmental Control Subsystem," NASA TND-6724, 1972.

${ }^{6}$ Jaax, J. R., Melgares, M. A., and Frahm, J. P., "Thermodynamic Performance Testing of the Orbiter Flash Evaporator System," Proceedings of the $11^{\text {th }}$ Space Simulation Conference, p. 43-54, 1980.

7 McQuillen, J., "Gas-Liquid Flows and Phase Separation," Strategic Research to Enable NASA's Exploration Missions Conference and Workshop, NASA/CP-2004-213205/VOL1, http://gltrs.grc.nasa.gov/reports/2004/CP-2004-213205-VOL1.pdf, pp $587-611,2004$.

${ }^{8}$ Lahey, R. T., and Dhir, V., "Research in Support of the Use of Rankine Cycle Energy Conversion Systems for Space Power and Propulsion," NASA/CR-2004-213142, http://gltrs.grc.nasa.gov/reports/2004/CR-2004-213142.pdf, 2004.

${ }^{9}$ Mudawar, I., Zhang, H., and Hasan, M. M., "Flow Boiling Critical heat Flux in Reduced Gravity," Strategic Research to Enable NASA's Exploration Missions Conference and Workshop, NASA/CP-2004-213205/VOL1, http://gltrs.grc.nasa.gov/reports/2004/CP-2004-213205-VOL1.pdf, pp 710 - 737, 2004.

${ }^{10}$ Morren, W. E., "Gravity Sensitivity of a Resistojet Water Vaporizer," AIAA-93-2402, 1993.
} 